\title{
ESTUDO SOBRE NASCIDOS VIVOS EM MATERNIDADES \\ 1. PESO AO NASCER, SEXO, TIPO DE NASCIMENTO E FILIAÇÃO PREVIDENCIÁRIA DAS MÃES
}

\author{
Maria de Lourdes R. de Souza* \\ Ana Cristina d'Andretta Tanaka** \\ Arnaldo Augusto Franco de Siqueira** \\ Renato Martins Santana***
}

\begin{abstract}
SOUZA, M. de L. R. de et al. Estudo sobre nascidos vivos em maternidades. 1. Peso ao nascer, sexo, tipo de nascimento e filiação das mães. Rev. Saúde públ., S. Paulo, 22:489-93, 1988.

RESUMO: O peso ao nascer do recém-nascido é o resultado de diversos fatores (orgânicos, psíquicos e sociais) sobre o potencial genético do feto. É natural que sua distribuição seja diferente conforme as características da população. Desta maneira, pretendeu-se estudar o peso ao nascer dos recém-nascidos vivos, de 1978 e 1979, de duas grandes maternidades de Florianopolis, SC (Brasil), nas quais ocorrem 90\% dos partos da região, segundo sexo, tipo de nascimento e filiação previdenciária das mães. Observou-se que a média de peso ao nascer dos 18.491 recém-nascidos vivos estudados foi de $3.347,6 \mathrm{~g}$. Nessa população ocorreu $5,3 \%$ de baixo peso ao nascer e $11,1 \%$ de crianças com $4.000 \mathrm{~g}$ ou mais. As crianças do sexo masculino pesaram ao nascer mais que as do sexo feminino, sendo esta diferença estatisticamente significativa. O mesmo fato ocorreu com as crianças de nascimentos únicos e múltiplos, tendo os primeiros peso ao nascer maior que os segundos, sendo também esta diferença estatisticamente significativa. $O$ estudo da relação entre a filiação previdenciária das mães e o peso ao nascer das crianças mostrou que as mulheres da classe "indigente/serv. social" tiveram número significativamente maior de recém-nascidos de baixo peso do que os de outras categorias sociais. Esses dados mostram que a população estudada apresentou uma baixa incidencia de baixo peso ao nascer, com uma distribuição de peso ao nascer semelhante a de países adiantados.
\end{abstract}

UNITERMOS: Peso ao nascer. Baixo peso ao nascer. Fatores sexuais. Fatores sócio-econômicos.

\section{INTRODUÇÃO}

O peso do recém-nascido ao nascer é uma variável de grande importância em saúde pública. Essa importância decorre, em grande parte, do reconhecimento de sua forte associação com a mortalidade não só no período perinatal como infantil. Para Chase 6 , o peso ao nascer é a variável isolada que mais interfere nessa mortalidade. Durante a Investigação Interamericana de Mortalidade na Infância, Puffer e Serrano"1 e Siqueira ${ }^{12}$ verificaram, em São Paulo, que $60 \%$ dos obitos neonatais referiam-se a crianças nascidas com peso igual ou inferior a $2.500 \mathrm{~g}$. Siqueira ${ }^{12}$ estimou que $10 \%$ dos nascidos vivos pesavam $2.500 \mathrm{~g}$ a menos, de tal forma que o risco de morrer dessas crianças, comparado ao de crianças nascidas com peso superior a $2.500 \mathrm{~g}$ era cerca de 20 vezes maior. $\mathrm{Na}$ California, Puffer e Serrano"11 mostraram que o risco de morrer, no período neonatal, dos recém-nascidos de baixo peso era mais de 40 vezes maior que o das demais crianças.
$O$ interesse em se estudar o peso do recém-nascido ao nascer não se extingue na sua associação com mortalidade.

$\mathrm{Na}$ verdade, a própria associação com a mortalidade neonatal depende da presença de outras condiçð̃es, como muito bem mostraram, entre outros, Battaglia e Lubchenco ${ }^{2}$, ao incluir nessa relação a idade gestacional. Segundo esses autores, se bem que, de uma maneira geral, à medida que diminui o peso do recém-nascido ao nascer aumenta a mortalidade, esse aumento é tanto mais nítido quanto menor a idade gestacional. Assim, recém-nascidos de baixo peso têm tanto maior risco de morrer quanto menor a idade gestacional.

Porém, talvez o mais importante no estudo de peso do recém-nascido ao nascer seja a sua explicação. Para tanto, há que inicialmente descrever como estão se distribuindo, em nosso meio, os nascimentos segundo o peso.

Como o peso ao nascer representa o resultado da atuação de uma série grande de fatores de natureza diversa sobre o potencial genético do

\footnotetext{
* Departamento de Saúde Pública do Centro de Ciências da Saúde da Universidade Federal de Santa Catarina - Cidade Universitária - Trindade -88000 - Florianópolis, SC - Brasil.

** Departamento de Saúde Materno-Infantil da Faculdade de Saúde Pública da Universidade de São Paulo - Av. Dr. Arnaldo, 715 - 01255 - São Paulo, SP - Brasil.

*** Departamento de Toco-Ginecologia da Escola Paulista de Medicina - Rua Napoleão de Barros, 715 - 04024 - São Paulo, SP - Brasil.
} 
concepto, é natural que sua distribuição seja diferente em populaçס̃es distintas, vivendo cada uma delas em condiçðes peculiares.

Assim como existem diferenças importantes na distribuição dos pesos dos recém-nascidos entre países, o que decorre, em sua maior parte, de condiçōes de vida ou em outras palavras, de fatores socio-ambientais ou adquiridos, também no Brasil não é possível generalizar para as suas diferentes regioes ou realidades os conhecimentos até aqui disponíveis que são, na verdade, bastante esparsos.

Afora trabalhos que refletem casuísticas de determinados hospitais ou serviços, como os de Dorea ${ }^{7}$, Siqueira ${ }^{12}$, Siqueira e col $^{13}$, existem poucos (como o de Monteiro ${ }^{10}$ ) que buscaram caracterizar o peso do recém-nascido em termos populacionais, baseado em dados hospitalares.

Em decorrência do fato de mais de $90 \%$ dos partos hospitalares em Florianopolis ocorrerem nas duas maternidades da cidade, o estudo dos nascimentos hospitalares fica, assim, facilitado.

O presente trabalho, o primeiro de uma série relativa a nascimentos em hospitais em Florianópolis, faz uma descrição geral desses nascimentos, em especial no que diz respeito ao peso do recém-nascido, sexo e tipo de nascimento e filiação previdenciária das mães.

\section{POPULAÇÃO E METODOLOGIA}

Foram utilizados os dados referentes a todas as crianças nascidas vivas, nos anos de $1978 \mathrm{e}$ 1979, em duas maternidades de grande porte, de Florianopolis, Santa Catarina.

Nesses 2 anos nasceram, nessas maternidades, 18.491 crianças vivas.

Segundo os dados do Censo de $1980^{\circ}$ nasceram, entre 1/9/79 e 31/8/80, na Grande Florianópolis, 11.506 crianças vivas, sendo $4.653 \mathrm{em}$ Florianópolis. Do total de 18.491 nascidos vivos, aqui estudados, referentes aos anos de 1978 e $1979,8.410$ eram filhos de mulheres residentes no Município de Florianópolis, o que mostra que a grande maioria dos nascimentos da região realmente ocorre nas duas maternidades estudadas.

Além do sexo e peso do recém-nascido ao nascer foram coletados nos SAMES (Serviço de Arquivo Médico e Estatística), das duas maternidades, dados sobre as seguintes variáveis maternas: idade, número de partos anteriores, tipo de parto, tipo de anestesia, tipo de nascimento, permanềncia no hospital, filiação previdenciária* e local de residência.

Os dados foram processados para serem submetidos à computação eletrônica. Os resultados foram expressos na forma tabular.

Quanto à análise estatística, utilizou-se a análise de variância a um critério, modelo fixo para os casos de diferenças entre mais de duas médias, tendo-se recorrido aos contrastes de Scheffé para identificar essas diferenças. Nos casos de diferenças entre duas médias utilizou-se a estatística " $t$ "'.

A significância das associações entre variáveis qualitativas foi feita com o auxilio do teste do $\mathbf{X}^{2}$.

Os títulos das tabelas foram simplificados, mas referem-se ao total de nascidos vivos das duas maternidades, nos anos de 1978 e 1979.

Em várias tabelas o total de casos foi inferior a 18.491 em virtude de faltarem informaçסes específicas sobre determinadas variáveis em alguns casos. O número de vezes em que isso ocorreu foi muito pequeno, em geral não alcançando $0,5 \%$ do total de casos incluídos no estudo.

O nivel de significância para a rejeição da hipótese de nulidade foi de $1 \%$.

\section{RESULTADOS E DISCUSSÃO}

O peso médio do recém-nascido ao nascer foi de 3.347,6 gramas, valor bastante satisfatório, quando comparado a dados de outras regióes do Brasil $3,4,12$.

A Tabela 1 mostra que as proporções de nascimentos nas faixas de peso inferiores a $2.500 \mathrm{~g}$ da presente casuística foram muito semelhantes às descritas por Chamberlains", na Grã-Bretanha.

A proporção de recém-nascidos de baixo peso nas duas maternidades de Florianópolis foi de 5,3\%. Inferior mesmo ao valor descrito para a Grã-Bretanha ${ }^{5}(6,8 \%)$.

Fato semelhante ocorreu nas faixas superiores de peso, eis que mais de $\mathbf{4 0 \%}$ dos nascimentos aqui referidos pesaram $3.500 \mathrm{~g}$ e mais. $\mathrm{Na}$ Grã-Bretanhas ${ }^{5}$ esse valor foi de $35 \%$ e na casuística de Laurenti e cols. ${ }^{9}$ não alcançou os $30 \%$.

Quanto à distribuição do peso segundo o sexo (Tabela 2), o peso médio dos recém-nascidos do sexo masculino $(3410,7 \mathrm{~g})$ foi significativa-

\footnotetext{
* Entende-se por filiação previdenciária a forma de pagamento pelos serviços de saúde recebidos, tendo sido as mulheres subdivididas em três grupos:

1. classe: particulares, beneficiários do Banco do Brasil, Eletrosul, Golden Cross, FUNSELF, Patronal e Gboex.

2. classe: INPS E IPESC

3. classe: Indigentes/serviço social.
} 
TABELA 1

Peso ao nascer. Comparação com dados de Laurenti e Cols. ${ }^{9}$ e Chamberlain ${ }^{5}$.

\begin{tabular}{lrrrrrr}
\hline \multicolumn{1}{c}{$\begin{array}{c}\text { Peso } \\
\text { ao } \\
\text { nascer (g) }\end{array}$} & \multicolumn{2}{c}{ Florianópolis } & \multicolumn{2}{c}{ Laurenti* } & \multicolumn{2}{c}{ Chamberlain** } \\
\hline No & $\%$ & N & \multicolumn{1}{c}{$\%$} & $\%$ & No & $\%$ \\
\hline $1500-1999$ & 117 & 0,6 & 187 & 1,5 & 177 & 1,0 \\
$2000-2499$ & 168 & 0,9 & 276 & 2,2 & 207 & 1,2 \\
$2500-2999$ & 697 & 3,8 & 946 & 7,4 & 781 & 4,6 \\
$3000-3499$ & 2.833 & 15,3 & 3.263 & 25,5 & 3.171 & 18,9 \\
$3500-3999$ & 7.120 & 38,5 & 4.884 & 38,2 & 6.564 & 39,0 \\
4000 e mais & 5.512 & 29,8 & 2.577 & 20,2 & 4.498 & 26,7 \\
s/inf & 1.959 & 10,6 & 585 & 9,6 & 1.386 & 8,2 \\
\hline Total & 85 & 0,5 & 64 & 0,5 & 31 & 0,2 \\
\hline
\end{tabular}

Fonte: * Laurenti e Cols. ${ }^{9}$

** Chamberlain ${ }^{5}$

TABELA 2

Peso dos nascidos vivos segundo o sexo. Florianópolis, 1978-1979

\begin{tabular}{llll}
\hline \multicolumn{1}{c}{ Sexo } & No & $\begin{array}{c}\text { média de } \\
\text { peso }(\mathrm{g})\end{array}$ & $\begin{array}{l}\text { Desvio } \\
\text { padrão }\end{array}$ \\
\hline masculino & 9.575 & $3.410,7$ & 560,46 \\
feminino & 8.831 & $3.279,1$ & 529,88 \\
\hline$t$ obs $=16,34$ & & & \\
$t$ crit $=2,58$ & $\mathrm{p}<0,01$ &
\end{tabular}

mente maior que o dos do sexo feminino $(3279,1 \mathrm{~g})$, de acordo, aliás, com o observado por Areno', por Siqueira e col. ${ }^{13}$ e por Tana$\mathrm{ka}^{14}$.

Quanto ao tipo de nascimento (Tabela 3) verificou-se, conforme era esperado, que os nascimentos gemelares tiveram um peso médio ao nascer de $2.523,0 \mathrm{~g}$, significativamente menor que os nascimentos únicos $(3.358,7 \mathrm{~g})$.

\section{TABELA 3}

Peso do nascido vivo (n.v.) segundo tipo de nascimento. Florianópolis, 1978 e 1979

\begin{tabular}{lccc}
\hline Tipo nasc. & $\begin{array}{c}\text { Nọ } \\
\text { casos }\end{array}$ & $\begin{array}{c}\text { Média de peso } \\
\text { n.v. (9) }\end{array}$ & $\begin{array}{c}\text { Desvio } \\
\text { padrão }\end{array}$ \\
\hline Onico & 18.175 & $3.358,7$ & 539,75 \\
Duplo & 242 & $2.523,0$ & 697,55 \\
\hline
\end{tabular}

t obs $=23,82$

t crit $=2,58 \quad p<0,01$
Esse fato pode ser melhor observado ao se proceder à divisão dos nascimentos por faixas de peso (Tabela 4). A tabela mostrou, pois, que apenas $4,7 \%$ dos nascimentos únicos pesaram menos de $2.500 \mathrm{~g}$, ao passo que $45,4 \%$ dos gemelares pesaram menos de $2.500 \mathrm{~g}$.

Ao se estudar a relação entre a filiação previdenciária das mães e o peso médio do recém-nascido vivo (Tabela 5), pode-se observar que a média de peso ao nascer das crianças, filhos de mulheres pertencentes à primeira classe era maior do que a daquelas pertencentes à segunda classe, e esta era maior, por sua vez, que as dos filhos das mulheres da classe "indigentes/serviço social".

\section{TABELA 4}

Distribuição do peso ao nascer dos nascidos vivos segundo tipo de nascimento. Florianópolis, 1978 e 1979

\begin{tabular}{|c|c|c|c|c|}
\hline \multirow{3}{*}{$\begin{array}{c}\text { Peso } \\
\text { ao } \\
\text { nascer }\end{array}$} & \multicolumn{4}{|c|}{ Tipo de Nascimento } \\
\hline & & & G & eos \\
\hline & No & $\%$ & No & $\%$ \\
\hline $\begin{array}{l}\text { até } 1499 \\
1500-1999 \\
2000-2499 \\
2500-2999 \\
3000-3499 \\
3500-3999 \\
4000 \text { g e mais }\end{array}$ & $\begin{array}{r}99 \\
151 \\
623 \\
2.752 \\
7.088 \\
5.508 \\
1.954\end{array}$ & $\begin{array}{r}0,5 \\
0,8 \\
3,4 \\
15,1 \\
39,0 \\
30,3 \\
10,8\end{array}$ & $\begin{array}{r}18 \\
18 \\
74 \\
83 \\
36 \\
6 \\
7\end{array}$ & $\begin{array}{r}7,4 \\
7,4 \\
30,6 \\
34,3 \\
14,9 \\
2,5 \\
2,9\end{array}$ \\
\hline Total* & 18.175 & 100,0 & 242 & 100,0 \\
\hline
\end{tabular}

- Em 74 casos não houve informação sobre tipo de nascimento e/ou peso ao nascer.

$$
\begin{aligned}
& \mathrm{X}^{2} \text { obs }=930,51728 \\
& \mathrm{X}^{2} \text { crit }=26,1
\end{aligned} \quad \mathrm{p}<0,01
$$




\section{TABELA 5}

Média de peso ao nascer segundo filiação previdenciária. Florianópolis, 1978 e 1979.

\begin{tabular}{lrcc}
\hline $\begin{array}{c}\text { Filiação } \\
\text { Previdenciária }\end{array}$ & $\begin{array}{c}\text { No } \\
\text { Casos }\end{array}$ & $\begin{array}{c}\text { Peso Médio } \\
\text { do n.v. (g) }\end{array}$ & $\begin{array}{c}\text { Desvio } \\
\text { padrão (g) }\end{array}$ \\
\hline Primeira classe & 547 & $3.389,6$ & 521,783 \\
Segunda classe & 16.207 & $3.366,4$ & 545,429 \\
"Indig/Ser. Soc." & 1.661 & $3.152,0$ & 568,811 \\
\hline Total & $18.415^{*}$ & $3.347,8$ & 550,344 \\
\hline
\end{tabular}

* 76 casos em que se ignora a filiação previdenciária.

$\mathrm{F}$ obs $=117,5$

$F_{\text {crit }}=4,61$

$\mathrm{p}<0,01$
Foram aplicados, a seguir, os contrastes de Scheffé, que revelaram que o peso médio dos nascidos vivos de mães "indigentes/ser. social" foi significativamente menor que o dos filhos das demais mulheres, ao nivel de $1 \%$.

Estudou-se a relação entre categorias de peso ao nascer e a filiação previdenciária. Devido ao pequeno número de casos em algumas categorias, optou-se por reunir numa categoria os recém-nascidos de peso inferior a $2.500 \mathrm{~g}$ e. de 3.000 a $3.999 \mathrm{~g}$ (Tabela 6).

$\mathrm{Na}$ Tabela 6, chamou a atenção o fato das mulheres da classe "indigentes/ser. social" terem apresentado mais do triplo de recém-nascidos de baixo peso que as da "primeira classe".

TABELA 6

Categorias de peso ao nascer segundo filiação previdenciária das mães. Florianópolis, 1978 a 1979.

\begin{tabular}{|c|c|c|c|c|c|c|c|c|}
\hline \multirow{3}{*}{$\begin{array}{c}\text { Peso } \\
\text { ao } \\
\text { nascer (g) }\end{array}$} & \multicolumn{8}{|c|}{ Filiaçāo Previdenciária } \\
\hline & \multicolumn{2}{|c|}{ Primeira Classe } & \multicolumn{2}{|c|}{ Segunda Classe } & \multicolumn{2}{|c|}{ Terceira Classe } & \multicolumn{2}{|c|}{ Total } \\
\hline & n? & $\%$ & n! & $\%$ & n? & $\%$ & n! & $\%$ \\
\hline $\begin{array}{l}<2.500 \\
2.500-2.999 \\
3.000-3.999 \\
4.000 \mathrm{e}^{+}\end{array}$ & $\begin{array}{r}17 \\
81 \\
393 \\
56\end{array}$ & $\begin{array}{r}3,1 \\
14,8 \\
71,8 \\
10,2\end{array}$ & $\begin{array}{r}807 \\
2.366 \\
11.223 \\
1.811\end{array}$ & $\begin{array}{r}5,0 \\
14,6 \\
69,2 \\
11,2\end{array}$ & $\begin{array}{r}158 \\
387 \\
1.022 \\
94\end{array}$ & $\begin{array}{r}9,5 \\
23,3 \\
61,5 \\
5,7\end{array}$ & $\begin{array}{r}982 \\
2.834 \\
12.638 \\
1.961\end{array}$ & $\begin{array}{r}5,3 \\
15,4 \\
68,6 \\
10,6\end{array}$ \\
\hline Total & 547 & 100,0 & 16.207 & 100,0 & 1.661 & 100,0 & 18.415 & 100,0 \\
\hline
\end{tabular}

$\mathrm{X}^{2}$ obs $=194,57$

$\mathrm{X}^{2}$ crit $=16,81$

$$
\mathbf{p}<0,01
$$

Além disso, dentre as mulheres desta classe, apenas $17,9 \%$ dos nascidos vivos apresentaram peso inferior a $3.000 \mathrm{~g}$; proporção semelhante foi verificada em relação aos filhos de mulheres da "segunda classe" $(19,6 \%)$; porém quase $1 / 3$ $(32,8 \%)$ dos filhos das mulheres da categoria "indigentes/ser. social" pesaram menos de $3.000 \mathrm{~g}$. Assim, não apenas a proporção de recém-nascidos de baixo peso mas também a de crianças de pesos de 2.500 a $\mathbf{2 . 9 9 9}$ gramas foi consideravelmente maior nesse grupo.

\section{COMENTÁRIOS}

Como já foi visto, a casuística aqui apresentada provavelmente está englobando a grande maioria dos nascimentos de Florianópolis e a quase totalidade dos nascimentos hospitalares.

A julgar pelos resultados, a proporção de nascimentos de crianças de peso inferior a $2.500 \mathrm{~g}$ é pequena, semelhante à que se verifica em países adiantados, muito diferente, aliás, dos resultados obtidos em nove maternidades-escola (incluindo uma das aqui estudadas), por Laurenti e col. ${ }^{9}$ Isto se explica pelo fato de aqui estarem representados todos os estratos da população, enquanto que a parcela da população atendida em hospitais-escola continua a ser constituida de gestantes de baixa renda, com baixa cobertura de pré-natal, com incidência aumentada de patologia da gestação, em suma, todos esses fatores que influem negativamente no peso do recém-nascido.

Além disso, sete das nove maternidades do citado estudo ${ }^{9}$ estão no Estado de São Paulo, onde é sabido que a incidência de baixo peso é maior, pelas próprias características da população ali assistida.

No entanto, ao se levar em conta a forma de pagamento dos serviços recebidos, a incidência de recém-nascidos de baixo peso e de peso insuficiente, dentre as gestantes não pagantes nem beneficiárias de quaisquer formas de seguro-saúde (chamadas de "indigentes/ser. social" neste trabalho), essas taxas se elevaram consideravelmente, mostrando que a população que apresentava dificuldades de acesso aos serviços de saúde era a que apresentava o perfil de peso ao nascer mais desfavorável. 
Os resultados permitem sugerir às autoridades sanitárias do Estado de Santa Catarina, ao invés de adotar propostas de intervenção direcionadas para a melhoria do peso do recém-nascido, dirijam suas atençð̃es para a ques- tão do acesso aos serviços de saúde à população menos favorecida, bem como para a avaliação e melhoria da qualidade da assistência pré-natal, ao parto e ao recém-nascido.

SOUZA, M. de L. R. de et al. [A study of life births in maternity hospitals. 1. Birth weight, sex, litter size and mothers' health security]. Rev. Saúde públ., S. Paulo, 22:489-93, 1988.

\begin{abstract}
Birthweight is the result of many factors (organic, psychological, social) acting on the genetic potential of the fetus. Consequently, its distribution is different according to the characteristics of the population. In this paper the authors studied the weight at birth of live newborns (from 1978 to 1979) in the two big maternity hospitals, in Florianopolis, responsible for $90 \%$ of all births in the area, by sex, litter size and mother's health security. The authors verified that the mean birthweight of the 18,491 live newborns was $3,347.6 \mathrm{gr}$. In that population $5.3 \%$ of the newborns were low birthweight infants, and $11.1 \%$ weighed $4,000 \mathrm{gr}$ or more. Male newborns weighed significantly more than female babies, and the same difference occurred between single and multiple births. The relationship between the newborn's birthweight and the kind of health security the mother had showed that the mothers who didn't pay any kind of health security had babies with lower birthweight than those of the mothers who subscribed to some health security scheme. The data showed that this population has a low incidence of low birthweight babies, with a distribution similar to that observed in developed countries.
\end{abstract}

UNITERMS: Birth weight. Infant, low birth weight. Sex factors. Socioeconomic factors.

\title{
REFERÊNCIAS BIBLIOGRÁFICAS
}

1. ARENO, F.B. Contribuição ao estudo da antropometria do recém-nascido. São Paulo, 1984. [Dissertação de Mestrado - Faculdade de Saúde Pública da USP].

2. BATTAGLIA, F.C. \& LUBCHENCO, L.O. A practical classification of newborn infants by weight and gestational age. J. Pediat., 71:159-63, 1967.

3. BENICIO, M.H.D'A. Fatores de risco de baixo peso ao nascer em recém-nascidos vivos - Município de São Paulo, 1978. São Paulo, 1983. [Tese de Doutoramento - Faculdade de Medicina da USP].

4. BÖELL, C.P. Peso ao nascer e sua relação com variáveis maternas em uma maternidade de Florianópolis - Santa Catarina. São Paulo, 1980. [Dissertação de Mestrado - Faculdade de Saúde Pública da USP].

5. CHAMBERLAIN, G. et al. British births 1970. London, William Heinemann Medical Books, 1975. v. 1.

6. CHASE, H.C. International comparisons of perinatal and infant mortality: the United States and Six West European Countries. Vital Hlth Statist. Ser. 3(6), 1967.

7. DOREA, A.D.R. Peso ao nascer de nascidos vivos na Maternidade do Hospital Universitário-Maceió-AL. São Paulo, 1981. [Dissettação de Mestrado - Faculdade de Saúde Pública da USP].

8. FUNDAÇÃO IBGE. Tabulaçסes avançadas do censo demográfico: resultados preliminares. Rio de Janeiro, 1981 .
9. LAURENTI, R. et al. Estudo da morbidade e da mortalidade perinatal em maternidades. I - Descrição do projeto e resultados gerais. Rev. Saúde públ., S. Paulo, 18:436-47, 1984.

10. MONTEIRO, C.A. O peso ao nascer no município de São Paulo: impacto sobre os níveis de mortalidade na infância. São Paulo, 1979. [Tese de Doutoramento - Faculdade de Saúde Pública da USP].

11. PUFFER, R.R. \& SERRANO, C.V. Patterns of mortolity in childhood. Washington, D.C., Pan American Health Organization, 1973. (PAHO - Scient. publ., 262).

12. SIQUEIRA, A.A.F. de Mortalidade neonatal e prematuridade. Sảo Paulo, 1974. [Dissertação de Mestrado - Faculdade de Saúde Pública da USP].

13. SIQUEIRA, A.A.F. de et al. Relação entre peso ao nascer, sexo e tipo de parto. Rev. Saúde públ., S. Paulo, 15:283-90, 1981.

14. TANAKA, A.C.d'A. Saúde materna e saúde perinatal: relaçōes entre variáveis orgânicas, sócio-econômicas e institucionais. São Paulo, 1986. [Tese de Doutoramento - Faculdade de Saúde Pública da USP].

Recebido para publicação em $28 / 1 / 1988$. Reapresentado em 13/9/1988.

Aprovado para publicação em 20/9/1988. 